Tropical Journal of Pharmaceutical Research April 2013; 12 (2): 163-168

ISSN: $1596-5996$ (print); 1596-9827 (electronic)

(C) Pharmacotherapy Group, Faculty of Pharmacy, University of Benin, Benin City, 300001 Nigeria.

All rights reserved.

Available online at http://www.tjpr.org

Original Research Article

http://dx.doi.org/10.4314/tjpr.v12i2.5

\title{
Development and Evaluation of Praziquantel Solid Dispersions in Sodium Starch Glycolate
}

\author{
Marco V Chaud ${ }^{1^{*}}$, Andréa C Lima ${ }^{2}$, Marta MDC Vila ${ }^{1}$, Maria O Paganelli ${ }^{3}$, Fábio C \\ Paula $^{4}$, Liliane N Pedreiro ${ }^{4}$ and Maria PD Gremião ${ }^{5}$ \\ ${ }^{1}$ Laboratory for Development and Evaluation of Bioactive Substances, Sorocaba University (UNISO), Sorocaba, SP, \\ ${ }^{2}$ Piracicaba Methodist University, Piracicaba, SP, ${ }^{3}$ Campinas University, UNICAMP. Campinas, SP, ${ }^{4}$ Ribeirão Preto University \\ (UNAERP), Ribeirão Preto-SP, ${ }^{5}$ São Paulo State University, UNESP. Araraquara, SP, Brazil
}

*For correspondence: E-mail::marco.chaud@prof.uniso.br; Tel: +55 (15) 2101-7104

Received: 29 May 2012

Revised accepted: 27 January 2013

\begin{abstract}
Purpose: To develop and characterize solid dispersions of praziquantel (PZQ) with sodium starch glycolate (SSG) for enhanced drug solubility.

Methods: PZQ solid dispersion (SD) was prepared using co-precipitation method by solvent evaporation. The ratios of PZQ to SSG were 2:1, 1:1, 1:2, 1:3 (w/w). PZQ solubility was evaluated in purified water, and $P Z Q$ dissolution test was carried out in $0.1 \mathrm{~N} \mathrm{HCl}$. Structural characterization of the dispersions was accomplished by $x$-ray diffraction (XRD) and infrared spectroscopy (FTIR) while the external morphology of the SDs, SSG and PZQ were studied by scanning electron microscopy (SEM). Mucoadhesion properties of the SD (1:3) and SSG, on mucin disks were examined using texture profile analysis.

Results: The highest solubility was obtained with $1: 3$ solid dispersion, with $P Z Q$ solubility of $97.31 \%$, which is 3.65-fold greater than the solubility of pure $P Z Q$ and physical misture $(P M, 1: 3)$. XRD results indicate a reduction in $P Z Q$ crystallinity while infrared spectra showed that the functional groups of $P Z Q$ and SSG were preserved. SEM showed that the physical structure of $P Z Q$ was modified from crystalline to amorphous. The amount of $P Z Q$ in $P M$ and $S D(1: 3)$ that dissolved in 60 min was 70 and $88 \%$, respectively, and these values increased to 76 and $96 \%$, respectively. The solid dispersion reduced the mucoadhesive property of the glycolate.

Conclusion: Solid dispersion formulation using SSG is a good alternative approach for increasing the dissolution rate of $P Z Q$.
\end{abstract}

Keywords: Praziquantel, Drug bioavailability, Schistosomiasis, Solid dispersion, Co-precipitation, Sodium starch glycolate

Tropical Journal of Pharmaceutical Research is indexed by Science Citation Index (SciSearch), Scopus, International Pharmaceutical Abstract, Chemical Abstracts, Embase, Index Copernicus, EBSCO, African Index Medicus, JournalSeek, Journal Citation Reports/Science Edition, Directory of Open Access Journals (DOAJ), African Journal Online, Bioline International, Open-J-Gate and Pharmacy Abstracts

\section{INTRODUCTION}

Poor bioavailability exerts strong limits to the performance of a drug by the necessity to administer a much higher dose than strictly required from a pharmacologic point of view. For good oral bioavailability drug must be soluble in gastrointestinal fluids, i.e., aqueous soluble and possess permeability properties for good membrane diffusion in order to reach the bloodstream [1].

Oral bioavailability of poorly soluble and highly permeable drugs, whose absorption is controlled by dissolution rate, can be increased by formulation strategies aimed at increasing both 
solubility and dissolution rate. Among the several strategies available for improving drug bioavailability, solid dispersions (SD) have been used extensively [2-6]. The SD technique produces a significant increase in surface area and surface wettability as well as solid state modification from crystalline to amorphous form. However, the SD characteristics can be influenced by several factors such as preparation method, carrier type and drug:carrier ratio and $\mathrm{pH}$ modifiers [7, 8].

Praziquantel (PZQ) is the first drug of choice in human taeniasis and schistosomiasis treatment. It is classified in Group II of Biopharmaceutical Classification System (BCS) and represents an example of a drug that requires research to improve drug solubility and reduce the high doses used in parasitic disease treatment. Funding of mass chemotherapy programs aimed at reducing the high morbidity rates associated to schistosomiasis is required [9]. SSG is the sodium salt of a carboxymethyl ether of starch, whose molecular weight is typically 500,000 $11,000,000$, and is widely used in oral pharmaceuticals as a disintegrant in capsule and tablet formulations. Sodium starch glycolate (SSG) is a very fine, white or off white, free flowing powder; odorless or almost odorless, practically insoluble in water and similarly insoluble in most organic solvents. Solid dispersion of carbamazepine with SSG increased solubility of carbamazepine due to the properties of wetting, significant increase in surface area and control over the particle size of the drug. Solid dispersions of SSG with ibersartan, olanzapine itraconazol, carbamazepina, nalidixic acid, furosemide $[2,10]$ produced higher dissolution rates than with other excipients due probably to their rapid dispersibility in the aqueous dissolution fluids [11-13]. The objective of this study was to investigate the dissolution rate of PZQ from solid dispersions (SD) prepared with SSG and also to structurally characterize the SD by $x$-ray diffraction (XRD), Fourier transformed infrared spectroscopy (FTIR) and scanning electron microscopy (SEM). The mucoadhesive performance of SD was also evaluated in vitro.

\section{EXPERIMENTAL}

\section{Materials}

Praziquantel (PZQ) with a purity of $99.6 \%$ was purchased from Indukern do Brasil Quimica Ltda (Brazil). Sodium starch glycolate was purchased from Blanver, Brazil. Other reagents used were of analytical grade.

\section{Preparation of solid dispersion}

PZQ and SSG were used at SD ratios of 2:1, 1:1, $1: 2$ and 1:3. A sufficient amount of ethanol was used to dissolve PZQ while SSG was dispersed in water. The mixture of PZQ solution with SSG dispersion was produced by mechanical agitation for $5 \mathrm{~min}$. When a clear solution was obtained, the sample was evaporated under reduced pressure at $65{ }^{\circ} \mathrm{C} \quad\left(\right.$ Tecnal ${ }^{\circledR}$ TE-210 rotary evaporator) and dried in a freeze-dryer (Edwards Modulyo ${ }^{\circledR}$ Freeze-Dryer). The co-precipitate (solid dispersion) was crushed in a porcelain mortar with a pestle and stored in a silica desiccator pending use. The viscosity of the SSG dispersion was measured (DV-E Brookfield viscometer) before mixing with the $P Z Q$ solution.

\section{Preparation of physical mixtures (PM) of PZQ and SSG}

PZQ and SSG powder mixtures (PM) at ratios of $2: 1,1: 1,1: 2$ and $1: 3$ were prepared by first sifting the individual powders through a $425 \mu \mathrm{m}$ aperture screen and mixing together by trituration in a mortar with a pestle. The mixture was stored in a silica desiccator pending use.

\section{Solubility studies}

The solubility of PZQ was determined in purified water. Briefly, $15 \mathrm{ml}$ of water was added to SD or $\mathrm{PM}$ (containing an equivalent of $15 \mathrm{mg}$ of $\mathrm{PZQ}$ ) in a test tube. The tube was sonicated $40 \mathrm{KHZ}$ for 30 min (Unique - Mod 2800A) and then shaken in a water bath $\left(25^{\circ} \mathrm{C}\right)$ for $48 \mathrm{~h}$. A portion of the solution $(10 \mathrm{ml})$ was withdrawn, centrifuged at $2,200 \mathrm{~g}$ for $20 \mathrm{~min}$, filtered $(0.45 \mu \mathrm{m}$ pore size) and centrifuged again at $7,000 \mathrm{~g}$ for $10 \mathrm{~min}$ twice. The upper portion $(1 \mathrm{ml})$ was then taken, diluted with $9 \mathrm{ml}$ of ethanol and the diluted solution subsequently and analyzed spectrophotometrically for PZQ content at $263 \mathrm{~nm}$.

\section{In vitro release studies}

The release rate of pure $P Z Q, P Z Q$ in $S D$ and in $P M$ was determined using a dissolution test (method II, USP 32). The dissolution test was performed in $900 \mathrm{~mL}$ of $0.1 \mathrm{M} \mathrm{HCl}$ with $0.2 \% \mathrm{w} / \mathrm{v}$ of sodium lauryl sulphate, at $37 \pm 0.5^{\circ} \mathrm{C}$, rotating at $50 \mathrm{rpm}$, for $120 \mathrm{~min}$. PZQ sample of $0.5 \mathrm{mg} \cdot \mathrm{mL}^{-1}$ were taken for dissolution studies at regular intervals. Withdrawing samples were filtered through a membrane filter (Allcrom, 0.22 $\mu \mathrm{m}$ pore size). The withdrawn sample, in each case, was replaced with an equivalent volume of 
fresh dissolution medium at $37{ }^{\circ} \mathrm{C}$. Absorbance of the samples was measured spectrophotometrically at $263 \mathrm{~nm}$.

\section{Scanning electron microscopy}

The external morphology of SD, PM and $\mathrm{PZQ}$ was studied by scanning electron microscopy (SEM). The samples were prepared for SEM by lightly sprinkling the powder onto a double-sided adhesive tape affixed to an aluminum stub. The stubs were then coated with gold to a thickness of about $300 \AA$ under an argon atmosphere using a gold sputter module in a high-vacuum evaporator. The samples were then randomly scanned and photomicrographs taken with a scanning electron microscope (JSM-6360).

\section{X-ray diffraction analysis}

Diffraction patterns (PXRD) of all the samples were obtained using X-ray diffraction on a $D$ 5000- Siemens device (filtered Ni, Cu.Ka radiation). The samples were scanned at increments of $0.02^{\circ}$ from $5^{\circ}$ to $40^{\circ}$ at a diffraction angle of $2 \theta$. The voltage and currents used were $40 \mathrm{kV}$ and $50 \mathrm{~mA}$.

\section{Fourier transform infrared spectroscopy}

The infrared spectra of pure $P Z Q$ and $S D, P M$ were obtained by the $\mathrm{KBr}$ disk method recorded on a Fourier transform infrared (FTIR) spectrophotometer (Perkin Elmer, Spectrum One). The spectra were obtained by scanning at b 400 to $4,000 \mathrm{~cm}^{-1}$ at a resolution of $2 \mathrm{~cm}^{-1}$. KBr pellets were prepared by gently mixing $1 \mathrm{mg}$ of the sample with $200 \mathrm{mg} \mathrm{KBr}$.

\section{Mucoadhesive test}

The mechanical properties of SD (1:3) and SSG were examined using texture profile analysis (TA.XT plus, Stable Micro Systems, UK) [14]. In the test, compressed mucin disc was affixed to a metal platform using double-sided adhesive tape and then affixed onto a cylindrical probe with double-sided adhesive tape. The mucin disc was hydrated with phosphate buffer $(\mathrm{pH} 7.4)$ for $60 \mathrm{~s}$. The test was performed in compression mode at a speed of $0.5 \mathrm{~mm} . \mathrm{s}^{-1}$ under a force of $2 \mathrm{mN}$. To come into contact with the mucin disc, the probe containing the sample was moved vertically at a speed of $2.0 \mathrm{~mm}^{-1} \mathrm{~s}^{-1}$. After $10 \mathrm{~min}$ of contact, the probe was moved in the opposite direction at the same speed. The maximum force required to separate the probe from the mucin disk was monitored by the in-built software (Texture Exponent Lite). The total force required (Wad) was calculated from the area of the plot of force versus distance. The analysis was performed in duplicate.

\section{Statistical analysis}

Two-way analysis of variance (ANOVA) and $t$ test were employed for statistical analysis of $\mathrm{PZQ}$ dissolution rate and solubility data. $P \leq 0.05$ was considered as indicative of statistically significant difference. The study was conducted using the software Microcal Origin v. 7.0 (OriginLab Corp).

\section{RESULTS}

The SD preparations were relatively simple and the SD mass was sufficiently friable to be ground easily.

\section{Solubility and in vitro release of PZQ}

Figure 1 shows $P Z Q$ solubility in purified water. Letters $a$ and $b$ compare the solubility of pure $P Z Q$ with $P Z Q$ in $P M$ or SD. Letters $c$ and $d$ compare the solubility of $P Z Q$ between the PMs and respective SDs. Equal letters indicate no statistical difference whereas different letters show a statistical difference for $p \leq 0.05$. In case of PMs, drug solubility seemed to decreased with increase in concentration of $P Z Q$, and none of them achieved the solubility of $\mathrm{PZQ}$ SDs. No statistical difference between the solubility of $P Z Q$ pure and the PMs was found. Moreover, a statistically significant difference was found between the solubility of $P Z Q$ pure and SDs as well as between physical mixtures and the SDs corresponding.

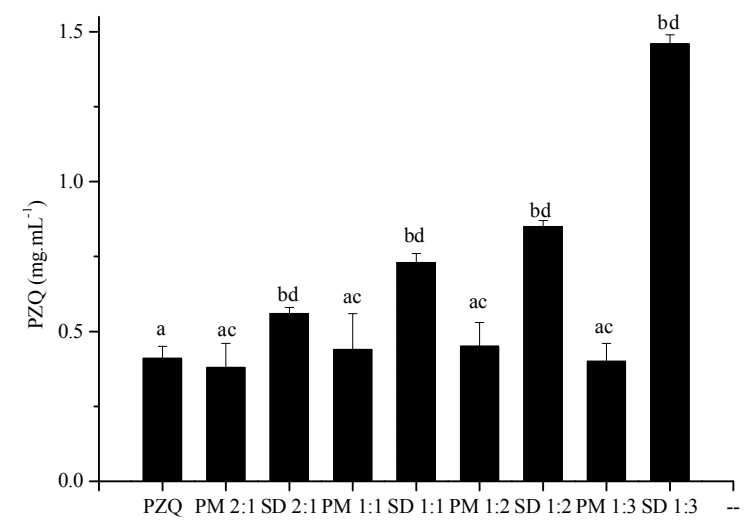

Figure 1: Solubility of pure $\mathrm{PZQ}, \mathrm{PM}$ and $\mathrm{SD}$ in water after $48 \mathrm{~h}$ of agitation. Letters $a$ and $b$ compare the solubility of pure $P Z Q$ with the solubility of $P Z Q$ in $P M$ and SD. Letters $c$ and $d$ compare the solubility of $P Z Q$ in $\mathrm{PM}$ and corresponding SD. Different letters represent statistically significant difference at $p \leq 0.05$. Data are expressed as mean $\pm S D(n=3)$.

Figure 2 shows the dissolution profile of pure $P Z Q$ and of $P Z Q$ in SD (1:3) and PM (1:3) 
formulations. Dissolved $\mathrm{PZQ}$ for $\mathrm{PM}$ and $\mathrm{SD}$ in $60 \min (P Z Q, P M \quad 1: 3)$ was 70 and $88 \%$, respectively. After $120 \mathrm{~min}$ (SD, 1:3), the figures increased to 76 and $96 \%$, respectively. Over the same time period (120 min.), dissolution of pure PZQ was only $64 \%$.

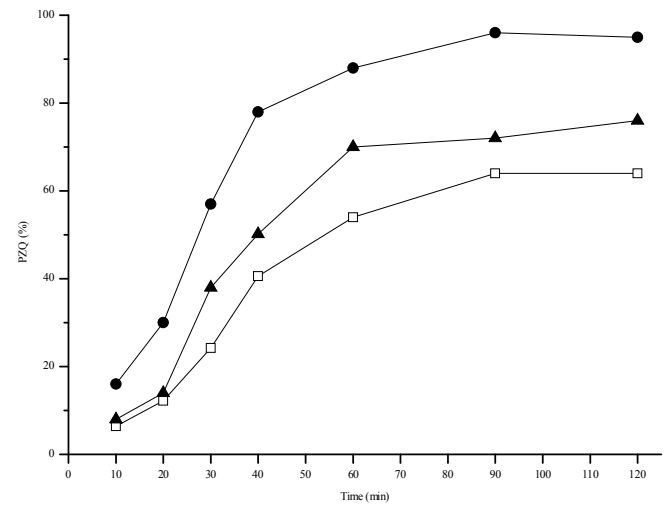

Figure 2: Dissolution profile of pure PZQ ( $\square$ ), 1:3 solid dispersion $(\bullet)$ and $1: 3$ physical mixture $(\boldsymbol{\Lambda})$. Data are expressed as mean $\pm S D(n=6)$

\section{Structural and molecular and morphological characterization of formulations}

The XRD diffractograms of SD, PM and the individual components are are shown in Fig 3. The polymer clearly exhibited an amorphous profile while $P Z Q$ was crystalline as evidenced by intense diffraction peaks). The diffraction pattern of PM was simply the superimposition of those of the individual components. On the other hand, the diffractogram of SD shows a change in the crystal structure of $P Z Q$, revealing a transition from a crystalline to an amorphous state.

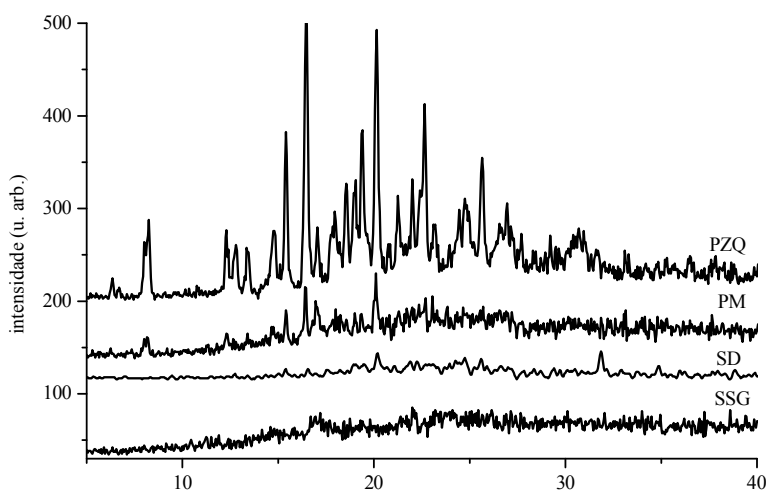

Figure 3: X-ray diffractohrams of pure PZQ, SSG, and $1: 3$ ratio of $P M$ and $S D$

The FTIR of pure PZQ, SD and PM are shown in Figure 4. For $P Z Q$, the wide band in the region 3600 - $3400 \mathrm{~cm}^{-1}$ indicate a characteristic stretching of the $\mathrm{O}-\mathrm{H}$ group which, in this case, can be attributed to the presence of water molecules. In the region $3000-2900 \mathrm{~cm}^{-1}$, vibration absorption bands of $\mathrm{C}-\mathrm{H}$ connections are seen, reflecting the symmetrical and asymmetric axial deformations of $\mathrm{CH}_{2}$ and $\mathrm{CH}_{3}$ groups. Stretching bands overlaid at $1651 \mathrm{~cm}^{-1}$ represent two carbonyls $(C=O)$ stretching absorption. At $1437 \mathrm{~cm}^{-1}, \mathrm{CH}_{2}$ angular stretching symmetrical bands appear with a band overlap partially observed at $1651 \mathrm{~cm}^{-1}$ for two connections stretching among carbons of the aromatic ring. Between 1350 and $1000 \mathrm{~cm}^{-1}$, axial deformation occurs with band overlapping of $\mathrm{C}-\mathrm{N}$ and $\mathrm{C}-\mathrm{H}$ symmetrical angular stretching of $\mathrm{CH}_{2}$. With regard to SSG spectrum (Figure 4B), a broad band in the region spanning $3600-2900$ reflects the $\mathrm{OH}$ stretching group of this compound molecule. At 1600 - $1000 \mathrm{~cm}^{-1}$, overlapping bands reflect asymmetric and symmetrical stretching of $\mathrm{C}-\mathrm{O}-\mathrm{C}$ group The scanning electron photomicrographs (SEM) of $P Z Q, S D$ and $S S G$ are displayed in Figure 5. Pure PZQ particles were crystalline (A) and SSG (B) spherical and non-porous, being oval or spherical granules, $30-100 \mu \mathrm{m}$ in diameter but with some in the size range of $10-35 \mu \mathrm{m}$ (diameter). SD (C) show structural change in the particles, with SSG particles losing their spherical shape and appear to increase volume. SD particles also presented filamentous shape and $P Z Q$ lost its original crystalline form.

\section{Mucoadhesive adhesive properties of solid dispersion}

SSG dispersed well in cold water and settled in the form of a highly hydrated layer; it exhibited a viscosity of $4.21 \mathrm{mPa}$. After mixing SSG dispersion with $\mathrm{PZQ}$ solution, the viscosity decreased to $3.36 \mathrm{mPa}$. The mucoadhesion of SSG and SD (1:3) was $1497 \pm 170$ and $1058 \pm$ $109 \mu \mathrm{J}$, respectively. The forces $(\mathrm{N})$ required to detach the formulations from mucin disc was $1735 \pm 0.888$ and $0.852 \pm 0.177$, respectively.

\section{DISCUSSION}

In this study, the solubility of PZQ in the SDs increased with increase in SSG concentration reaching a maximum at $P Z Q: S S G$ ratio of 1:3. This can be attributed to the swelling of SSG, which has a tendency to absorb water and retain it, resulting in up to $250 \%$ increase in particle diameter when exposed to water [15]. This volume increase in their particle diameter could serve as an impediment to drug release and could explain the decrease in drug solubility observed with an increase in their respective concentrations [16]. In this study, increase drug solubility may be due to significant reduction of 

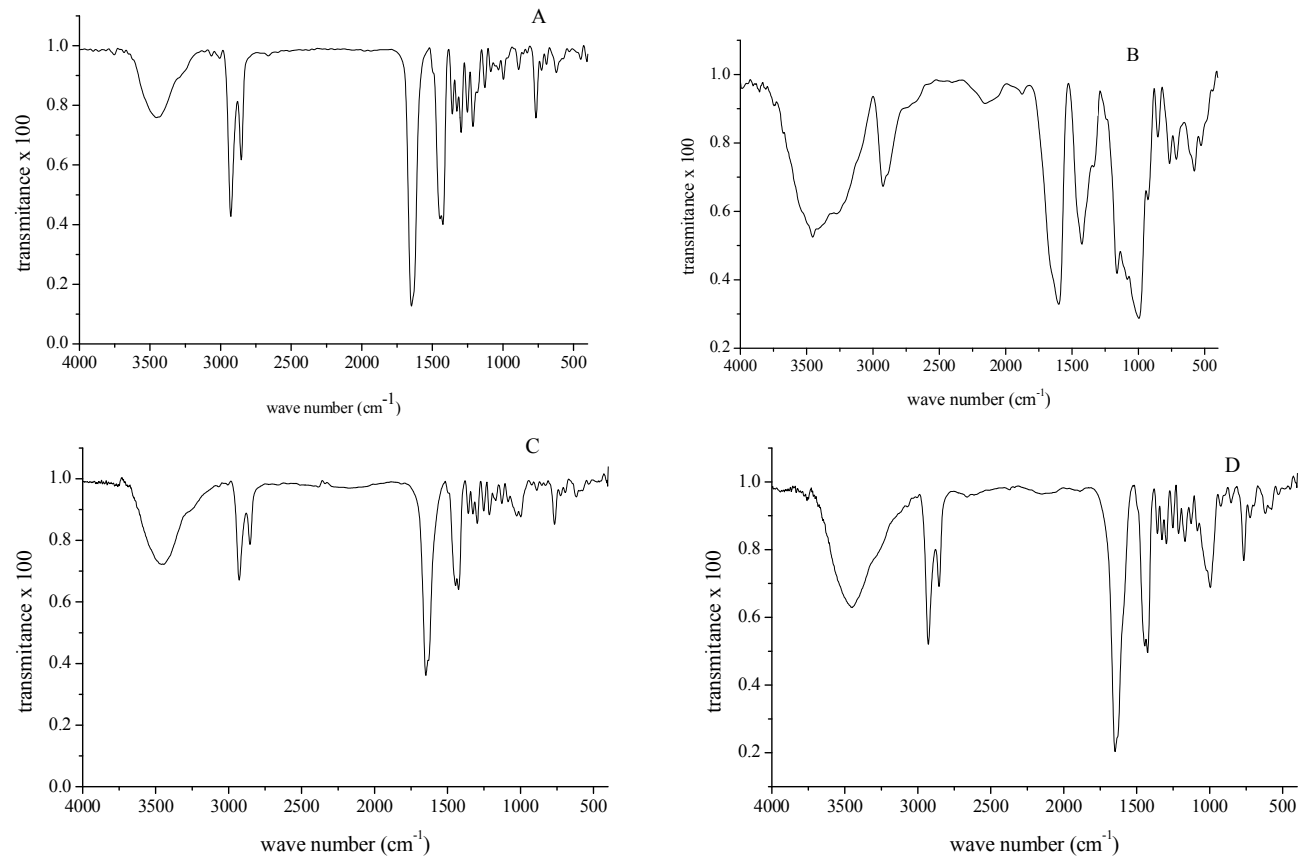

Figure 4: FTIR spectra of pure PZQ (A); SSG (B); 1:3 SD (C); and 1:3 PM (D)

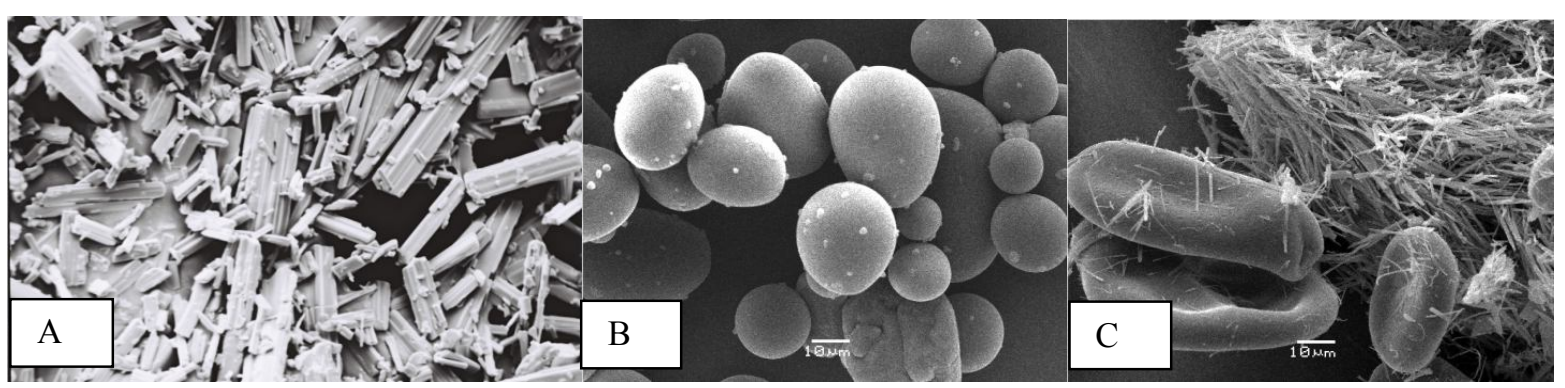

Figure 5: Scanning electron photomicrographs (x 1000) of pure PZQ (A); SSG (B) and SD 1:3 (C)

the crystallinity of the drug, as shown by x-ray diffraction results.

Increase in dissolution was partly dependent on reduction in drug crystallinity and is consistent with solubility results. The greater solubility of $P Z Q$ in $P M$ than that of the pure drug is an indication of increased drug wettability promoted by SSG. The results show that $P Z Q$ dissolution in SD was significantly greater than in PM. Enhancement of dissolution of $\mathrm{PZQ}$ from solid dispersion (SD) can be attributed to several factors which affect the mechanism of dissolution in solid dispersion. These include reduction of drug crystallinity, i.e., amorphization, increased wettability and dispersibility $[4,5,17]$.

$P Z Q$ is crystalline while SSG is amorphous. However,. This result helps explain the higher solubility and dissolution rate of $P Z Q$ in SD. The smaller diffraction peaks of SD compared with those of pure $P Z Q$ and $P Z Q$ in $P M$ can be attributed principally to the conversion of $P Z Q$ from a crystalline to amorphous state.
IR indicates that bonding, if present, might not have been strong as to suppress the spectral band of $P Z Q$. In both $S D$ and $P M$, the characteristic stretching of the functional drug groups was preserved, indicating the physical and chemical integrity of $P Z Q$ remained intact. It is therefore presumed that formation of solid dispersion or physical mixture does not cause any physical and/or chemical interaction between PZQ and SSG at molecular level.

Mucoadhesion is used to increase the residence time of drug at a specific site [20]. Generally, the polymer chains need to be mobile to interpenetrate the mucus glycoprotein chains and contribute to the consolidation of the mucoadhesive phenomenon. SSG, chemically described as the sodium salt of a carboxymethyl ether of starch, presents, in addition to hydroxyl group, only one cluster for ionization and interaction with mucin. Although $\mathrm{PZQ}$ easily permeates the intestinal mucosa, its bioavailability is limited by the rate of dissolution. However, mucoadhesion associated with 
increased solubility of $P Z Q$ in solid dispersion is a strategy that can result in improved bioavailability of $P Z Q$.

\section{CONCLUSION}

This study shows that the dissolution rate of $P Z Q$ can be enhanced greatly by the solid dispersion technique. The mucoadhesive ability of the SSG was confirmed for the SD 1:3 formulation. The results of this study, like those of a few others exploring solid dispersion strategy, indicate that solid dispersion is a viable solution for pharmaceutical companies to enhance the life cycle of the existing products in which poor solubility is a major concern. The SD of $P Z Q$ with mucoadhesive and hydrodispersible carriers also represents a useful strategy for increasing the bioavailability of poorly water-soluble drugs.

\section{ACKNOWLEDGMENT}

FAPESP (São Paulo Research Foundation) and Sorocaba University is gratefully acknowledged for supporting this work.

\section{REFERENCES}

1. Maheshwari RK, Jagwani Y: Mixed Hydrotropy: Novel Science of Solubility Enhancement. Indian J Pharm Sci 2011; 73(2): 179-183.

2. Yogesh $R$, Rajshree M, Mayur S, Jolly $S$, . Effect of hydrophilic swellable polymers on dissolution enhancement of Carbamazepine solid dispersions studied using response surface methodology. AAPS PharmSciTech 2007; 8(2): E1-E11.

3. Sun $Y$, Yang $R$, Zhou W, Tang $X$ : Nimodipine semi-solid capsules containing solid dispersion for improving dissolution. Int J Pharm 2008; 359: 144-149.

4. Chaud MV, Tamascia $P$, Lima AC, Paganelli $M O$ Gremião MPD, Freitas O: Solid dispersions with hydrogenated castor oil increase solubility, dissolution rate and intestinal absorption of praziquantel. Brazilian J Pharm Sci 2010; 46: 473481.
5. Correa EM, Vila MMDC, Junior JMO, Zaparoli RE, Granato MM, Goes AL, Moraes LC, Paula FC, Chaud MV: Assessment of solubility and intestinal absorption in vitro of praziquantel in solid dispersions of polyethylene glycol 6000. J Latin Am Pharm Sci 2011; 30: 1910-1915.

6. Sharma A, Jain CP: Preaparation and characterization of solid dispersion of carvedilol with PVP K30. Res Pharm Sci 2010; 5(1): 49-56.

7. Sethia S, Squillante E: Solid dispersions: revival with greater possibilites and applications in oral drug delivery. Critical Rev Ther Drug Carrier Systems 2003; 20: 215-247.

8. Tran T-D, Tran PH-L, Choi H-G, Han H-K, Lee B-J: The roles of acidifiers in solid dispersion and physical mixtures. Int J Pharm 2010; 384(1): 60-66.

9. Barsoum RS: Schistosomiasis and the kidney. Seminars in Nephrology 2003; 23(1): 34-41.

10. Chowdary KP, Rao SS: Investigation of dissolution enhancement of itraconazole by solid dispersion in superdisintegrants. Drug Development and Industrial Pharmacy 2000; 26: 1207-1211.

11. Krishnamoorthy $V$, Nagalingam $A$, Prasad VPR, Parameshwaran S, George $N$, Kaliyan $P$ : Characterization of Olanzapine-Solid Dispersions Iranian J Pharm Res 2011; 10(1): 13-24.

12. Meka AK, Pola S, Tupally KR, Abbaraju PL: Development, evaluation and characterization of surface solid dispersion for solubility and dissolution enhancement of Irbesartan. Int J Drug Dev Res 2012; 4(1): 263-273.

13. Ramana G, Jyothirmai $D$, Sri Vaishnavi $P$ : Sustained release of nipedipine from matrix tablets of its solid dispersion employing superdisintegrants. Int Res $J$ Pharm 2011, 2(9):166-169.

14. Franzén $N$, Björk E, Edsman $K$ : Changes in the mucoadhesion of powder formulations after drug application investigated with a simplified method. J Pham Sci 2008; 97: 3855-3864.

15. Balasubramaniam J, Tim B: The influence of superdesintegrant choice on the rate of drug dissolution. Pharm Technol 2009; 21: S1-S2.

16. Balasubramaniam J, Rajesh $Y$, Bindu $K$, Hemalatha $T$, Swetha M, Vinay UR: Enhanced dissolution and bioavailability of raloxifene hydrochloride by cogrinding with different superdisintegrants. Chem Pharm Bull 2010; 58: 293-300.

17. Chaulang G, Patel P, Hardikar S, Kelkar M, Bhosale A, Bhise B: Formulation and Evaluation of Solid Dispersions of Furosemide in Sodium Starch Glycolate. Trop J Pharm Res 2009 2009; 8(1): 4351. 